\title{
ON THE ORIGIN OF THE ANOMALOUS PRECESSION OF MERCURY'S PERIHELION
}

\author{
JAUME GINÉ
}

\begin{abstract}
Action at distance in Newtonian physics is replaced by finite propagation speeds in classical post-Newtonian physics. As a result, the differential equations of motion in Newtonian physics are replaced by functional differential equations, where the delay associated with the finite propagation speed is taken into account. Newtonian equations of motion, with post-Newtonian corrections, are often used to approximate the functional differential equations. In [12] a simple atomic model based on a functional differential equation which reproduces the quantized Bohr atomic model was presented. The unique assumption was that the electrodynamic interaction has a finite propagation speed. In 13 a simple gravitational model based on a functional differential equation which gives a gravitational quantification and an explanation of the modified Titius-Bode law is described. Firstly, in this work, we recall the calculations made by Einstein to arrive at the explanation of the anomalous precession of Mercury's perihelion. Secondly, we recover an ancient work of Gerber in 1898 as a precursor of the retarded theories, see [9]. In this paper Gerber gave an explanation of the anomalous precession of the Mercury's perihelion in terms of a velocity-dependent potential. In this paper an explanation of the anomalous precession of Mercury's perihelion is given in terms of a simple retarded potential, which, at first order, coincides with Gerber's potential, and which agrees with the author's previous works [12]13.
\end{abstract}

\section{INTRODUCTION}

The problem of the anomalous precession of the Mercury's perihelion appeared in 1859 when the French astronomer Le Verrier observed that the perihelion of the planet Mercury precesses at a slightly faster rate than can be accounted the Newtonian mechanics with the distribution

1991 Mathematics Subject Classification. Primary 34C05. Secondary 58F14.

Key words and phrases. gravitation, retarded systems, functional differential equations, limit cycle.

The author is partially supported by a DGICYT grant number BFM 2002-04236C02-01 and by DURSI of Government of Catalonia "Distinció de la Generalitat de Catalunya per a la promoció de la recerca universitària". 
of masses of the solar system well-known until then. This discovery began different lines of investigation to explain the new phenomenon. One of the explanations was the existence of a new planet that would explain the anomaly in Mercury's orbit within the context of Newton's laws. In others lines of investigation it was considered the modification or re-interpretation of the Newton's law of gravitation so that it would give Mercury's precession with the known distribution of masses of the solar system. For a complete description of the historical development of the problem see [16, 17].

Einstein found that the extra precession arises unavoidably from the fundamental principles of General Relativity. The general problem of the integration of the Einstein equations, given by

$$
R_{\mu \nu}-\frac{1}{2} R g_{\mu \nu}=\frac{8 \pi G}{c^{4}} T_{\mu \nu}
$$

where $R_{\mu \nu}$ is the Ricci tensor, $R$ is the Ricci scalar, $g_{\mu \nu}$ is the metric tensor and $T_{\mu \nu}$ is the stress-energy tensor, is extremely difficult and the determination of the explicit solutions is only possible in a restrict number of cases. One of the most important is the Schwarzschild solution for the case of a punctual mass or spherical and homogeneous and with the assumption that the limit values in the infinite of the $g_{\mu \nu}$ are the galilean values.

In [16] is given how to determine the relativistic prediction for the advance of an elliptical orbit from the Schwarzschild solution in a very comprehensible and clear form.

\section{GERBER'S THEORY}

At the end of the 19th century, theoretical physicists were investigating modifications of the Coulomb inverse-square law. For instance, Gauss and Weber introduced velocity-dependent potential to represent the electromagnetic field, consistent with the finite propagation speed of changes in the field. The application of this velocity-dependent potential to the gravitation was immediately. Several physicists propose different gravitational potentials based on finite propagation speed in order to account the Mercury's orbital precession, see for instance [19, 20, for a review of these proposals.

In fact, this line of investigation goes back to the works of Laplace [14] in 1805 where it is presented a correction of the Newton force produced by the particle $m_{1}$ in $m_{2}$, which moves with velocity $v$ given by

$$
\mathbf{F}=-G m_{1} m_{2}\left(\frac{\mathbf{r}}{r^{3}}+\frac{\mathbf{v}}{h}\right)
$$


where $h$ is the finite propagation speed. But this work didn't find echo practically until the surroundings of 1880 , when a series of works to estimate the gravitational finite propagation speed began. A brief list of authors that used the hypothesis of the finite propagation speed is Th. von Oppolzer (1883), J. von Hepperger (1889), R. Lehmann-Filhes (1894), K. Schwarzschild (1900), H. Minkowski (1908), H. Poincaré (1908), W. Ritz (1909). In other works different forms for the gravitational potential were proposed; we can mention H. von Seeliger (1895) and C. Neumann (1896). Under the influence of the electrodynamical development made by F. Neumann (1845), W. Weber (1846) and B. Riemann (1861), some authors began to think in modifying Newton's law adding terms which depend on the speeds of the involved bodies, see for instance [25]. In 1870 F.G. Holzmuller [8] proposed a law of gravitation of the same form that the electrodynamic Weber's law, given by

$$
F=\frac{G m_{1} m_{2}}{r^{2}}\left(1-\frac{\dot{r}^{2}}{h^{2}}+\frac{2 r \ddot{r}}{h^{2}}\right) .
$$

Later, F. Tisserand 24] had used this law to study the anomalous precession of Mercury's perihelion and he explained only 14.1 arc seconds per century. In the same way O. Liman (1886) and M. Lévi (1890), proposed a law of gravitation of the same form that the electrodynamic Riemann's law, given by

$$
F=\frac{G m_{1} m_{2}}{r^{2}}\left(1-\frac{\left(\dot{r}_{1}-\dot{r}_{2}\right)^{2}}{h^{2}}\right),
$$

where $r_{1}$ and $r_{2}$ are the position vectors of the particles $m_{1}$ and $m_{2}$, respectively. The Riemann-Liman-Lévi law explained only 28 arc seconds per century of the anomalous precession of Mercury's perihelion. Finally, M. Lévi, by means of a purely formal development, found a force law that led to the observed exact value of the anomalous precession of Mercury's perihelion. The theories to explain the form of the proposed law forces are based, in general, in to do a parallelism between the electromagnetism and the gravitation and to propose what is known as gravitational field with a gravelectric component and with a gravomagnetic component, see [1, 15] and references therein. Below, in the next section, we will see that all these laws are, in fact, developments until certain order of a retarded potential. These lines of research were abandoned when it was definitively implanted Einstein's Relativity theory. 
One of the first velocity-dependent potential used was

$$
V(r, \dot{r})=-\frac{m}{r} \frac{1}{\left(1-\frac{\dot{r}}{c}\right)},
$$

where it is incorporate a finite propagation speed into the law of gravity substituting the retarded radial distance for the present distance. This velocity-dependent potential predicts only one third of the observed value for the anomalous precession of Mercury's perihelion, see [17.

A German school teacher named Paul Gerber proposed in 1898 a velocity-dependent potential that predicts exactly the observed value for the anomalous precession of Mercury's perihelion, see [9, 10]. In [17] it is concluded with a speculative re-construction of a semi-classical line of reasoning by which it is actually possible to derive Gerber's potential, albeit in a way that evidently never occurred to Gerber. The proposed Gerber's velocity-dependent potential is

$$
V(r, \dot{r})=-\frac{m}{r} \frac{1}{\left(1-\frac{\dot{r}}{c}\right)^{2}} .
$$

which depends not only on the radial distance from the gravitational mass but also on the derivative (with respect to time) of that distance. The force law associated to this velocity-dependent potential is

$$
\begin{aligned}
f & =\frac{d}{d t}\left(\frac{\partial V}{\partial \dot{r}}\right)-\frac{\partial V}{\partial r} \\
& =-\frac{m}{r^{2}}\left(1-\frac{\dot{r}}{c}\right)^{-4}\left(\frac{6 r \ddot{r}}{c^{2}}-\frac{2 \dot{r}}{c}\left(1-\frac{\dot{r}}{c}\right)+\left(1-\frac{\dot{r}}{c}\right)^{2}\right) .
\end{aligned}
$$

and the expansion of this expression in powers of $\dot{r} / c$, gives

$$
f=-\frac{m}{r^{2}}\left(1-\frac{3 \dot{r}^{2}}{c^{2}}+\frac{6 r \ddot{r}}{c^{2}}-\frac{8 \dot{r}^{3}}{c^{3}}+\frac{24 r \dot{r} \ddot{r}}{c^{3}}-\ldots\right) .
$$

In [17], it is showed that the Gerber's velocity-dependent potential (11) results in elliptical orbits that precess by the same amounts as predicted by General Relativity (to the lowest order of approximation), and of course these fact agrees with the observed precession rates for the perihelia of the planets, including Mercury. The question, then, is whether we can justify the use of this particular velocity-dependent potential rather than the Newtonian potential $V=-m / r(t)$. Moreover, in [17] it is also showed that although General Relativity and Gerber's potential predict the same first-order precession, the respective equations of motion are not identical, even at the first non-Newtonian level of approximation. One of the objectives in the Gerber's works, taking 
into account the assumption of a finite propagation speed, was to infer the speed of gravity from observations of the solar system. The open question is if gravity and light move at the same speed, that it is still today on discussing, see [26] and references therein. In the introduction of the Gerber's paper 12, Ernst Gehrcke concludes:

Whether and how the theory of Gerber can be merged with the well-known electromagnetic equations into a new unified theory is a difficult problem, which still awaits a solution.

\section{A simple Retarded Potential}

Action at distance in Newtonian physics is replaced by finite propagation speeds in classical post-Newtonian physics. As a result, the differential equations of motion in Newtonian physics are replaced by functional differential equations, where the delay associated with the finite propagation speed is taken into account. Newtonian equations of motion, with post-Newtonian corrections, are often used to approximate the functional differential equations, see, for instance, [2, 3, 4, 15, 11, 22, 23. In [12 a simple atomic model based on a functional differential equation which reproduces the quantized Bohr atomic model was presented. The unique assumption was that the electrodynamic interaction has finite propagation speed, which is a consequence of the Relativity theory. An straightforward consequence of the theory developed in 12, and taking into account that gravitational interaction has also a finite propagation speed, is that the same model is applicable to the gravitational 2-body problem. In [13] a simple gravitational model based on a functional differential equation which gives a gravitational quantification and an explanation of the modified Titius-Bode law is described. In the following an explanation of the anomalous precession of Mercury's perihelion is given in terms of a simple retarded potential, which, at first order, coincides with the Gerber's potential.

The most straightforward way of incorporating a finite propagation speed into the law of gravity is to simplistically substitute the present distance for the retarded radial distance, therefore, we consider the simplest retarded potential

$$
V=-\frac{m}{r(t-\tau)}
$$

where $r(t)$ denotes the instantaneous position vector of the test particle, at time $t$, and $\tau$ is the delay, so that $r(t-\tau)$ is the retarded 
position of the test particle. In fact this retarded potential depends on the position vector but also on the velocity vector $\dot{r}$, on the acceleration vector $\ddot{r}$ an so on. The appearance of a delay implies all these dependences in the potential. From the retarded potential (3) we will obtain, in a theoretical point of view, the equation of motion of the particle. This equation will be a functional differential equation. The functional differential equations of motion are generally difficult, often impossible, to express in a form that is amenable to analysis. Thus, in order to obtain useful dynamical predictions from realistic models, it is frequent to replace the functional differential equations of motion by approximations that are ordinary or partial differential equations, see 2. In our case, if we develop the retarded potential (3) in powers of $\tau$ (up to second order in $\tau$ ), we obtain

$$
V \approx-\frac{m}{r}\left[1+\frac{\dot{r}}{r} \tau+\left(\frac{\dot{r}^{2}}{r^{2}}-\frac{\ddot{r}}{2 r}\right) \tau^{2}\right],
$$

To develop some easier calculations we can reject on the right hand side of expression (4) the term with $\ddot{r}$ (in fact this term is negligible and only gives terms of higher order). Hence, at this approximation, we obtain the velocity-dependent potential

$$
V \approx-\frac{m}{r}\left[1+\frac{\dot{r}}{r} \tau+\frac{\dot{r}^{2}}{r^{2}} \tau^{2}\right],
$$

In a first approximation, the delay $\tau$ must be equal to $r / c$ (the time that the field uses to go from Mercury to the Sun at the speed of the light) and according with the theories developed in [12, 13, we introduce a new constant $g$ in the delay and hence, $\tau=g r / c$. Introducing this expression of the delay in (6) we have

$$
V \approx-\frac{m}{r}\left[1+g \frac{r \dot{r}}{c r}+g^{2} \frac{r^{2} \dot{r}^{2}}{c^{2} r^{2}}\right] .
$$

On this basis, of this velocity-dependent potential function (6), the gravitational force law is given by substituting the potential function (6) into equation

$$
f=\frac{d}{d t}\left(\frac{\partial V}{\partial \dot{r}}\right)-\frac{\partial V}{\partial r}=-\frac{m}{r^{2}}\left(1-\frac{g^{2} \dot{r}^{2}}{c^{2}}+\frac{2 g^{2} r \ddot{r}}{c^{2}}\right) .
$$

It is easy to see that if we fix $g=\sqrt{3}$, we obtain the same radial force, at first orders, that gives Gerber's potential, see (2). In fact, we have constructed a potential that, varying $g$, predicts $2 g^{2} \pi m /\left(L c^{2}\right)$ as nonNewtonian advance of orbital perihelia per revolution, where $m$ is the Sun's mass, $L$ is the semi-latus rectum of the orbit, and $c$ is the speed of 
the light. Note that for $g=1$, it results in a value which is one third of the observed value, so it predicts only 14.1 arc seconds per century for the precession of Mercury's perihelion. The problem of the retarded potential (3) is that it can account for the anomalous precession of the Mercury's perihelion precisely by adjusting a free parameter of the theory. In the following we give a retarded potential which gives an explanation of the anomalous precession of the Mercury's perihelion without adjusting any free parameter of the theory. We will see that this new retarded potential also coincides, at first order, with Gerber's one.

We now consider a small modification of the retarded potential (3), given by

$$
V=-\frac{m}{r(t-\tau)} \frac{r(t)}{r(t-\tau)},
$$

where the modification consists on dividing the retarded potential (3) by the quotient $r(t-\tau) / r(t)$. And this quotient represents the ratio of the distance between the masses when the potential was "emitted" to distance between the masses at the present instant. We can think that the retarded potential (3) was obtained from the Newtonian potential $V=-m / r(t)$ of the form

$$
V=-\frac{m}{r(t) \frac{r(t-\tau)}{r(t)}}=-\frac{m}{r(t-\tau)},
$$

and the quotient $r(t-\tau) / r(t)$ is the corrective factor to obtain the retarded distance. This corrective factor is applied because the potential must propagate from the source to the location particle in question. In the same way we can think that the retarded potential (17) is obtained from the Newtonian potential $V=-m / r(t)$ of the form

$$
V=-\frac{m}{r(t) \frac{r(t-\tau)}{r(t)} \frac{r(t-\tau)}{r(t)}}=-\frac{m}{r(t-\tau)} \frac{r(t)}{r(t-\tau)},
$$

In the same way that in the Neumann's theories [18 we conceive the potential essentially as information being transmitted from place to place, and assumed a finite speed for the propagation of this information.

As it is written in [17, a particle sends forth a potential, the value of which depends not only on the emitting particle, but on the receiving particle. Therefore, the information must come back from the receiving particle to the emitting particle. Thus we ought to regard an elementary interaction not as a one-way exchange, but as a two-way 

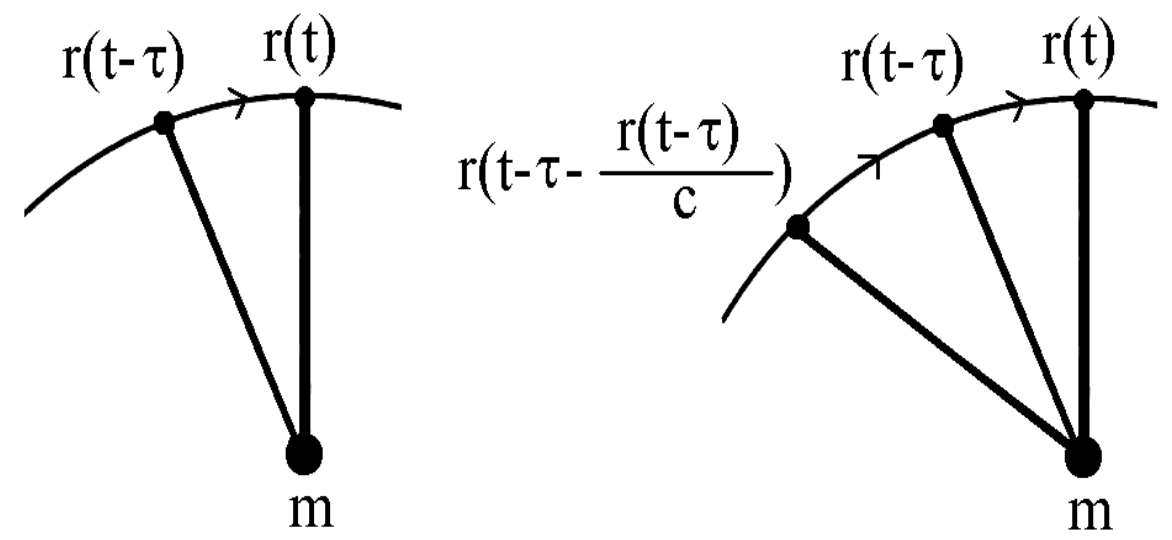

Figure 1. The retarded position of the test particle.

round-trip exchange. Hence, we must apply the corrective factor twice in the initial potential.

In fact the correct expression of the retarded potential, taking into account that the information must do a two-way round-trip and that $\tau=r(t) / c$, is

$$
V=-\frac{m}{r\left(t-\tau-\frac{r(t-\tau)}{c}\right)} .
$$

where $r(t-\tau-r(t-\tau) / c)$ is the distance between the masses when the potential was "emitted" to go from the emitting particle to the receiving particle and come back, see the second graphic of Fig. 1. To find the retarded potential (17) as approximation of the retarded potential (8) we take into account that for a small $\tau$ we have that

$$
r(t) r\left(t-\tau-\frac{r(t-\tau)}{c}\right) \approx(r(t-\tau))^{2} .
$$

Therefore, for a small $\tau$ we obtain

$$
V=-\frac{m}{r\left(t-\tau-\frac{r(t-\tau)}{c}\right)} \approx-\frac{m}{r(t-\tau)} \frac{r(t)}{r(t-\tau)} .
$$

Hence, the correct retarded potential is (8), but is a functional potential which is difficult to express in a form that is amenable to analysis. Therefore, we use the approximation (17) whose physical interpretation and use is totally justified. In fact, the retarded potential (71) is a generalization of the Gerber's potential. The Gerber's potential is the particular case when the velocity of the test particle is constant, i.e., 
when $\ddot{r}=0$. In [17] a physical explanation (albeit in a way that evidently never occurred to Gerber) of the form of the Gerber's potential is given.

Now we are going to see that the retarded potential (17) gives an explanation of the anomalous precession of Mercury's perihelion because coincides, at first order, with the force law associated to Gerber's one. If we develop the retarded potential (17) in powers of $\tau$ (up to second order in $\tau$ ), we obtain

$$
V \approx-\frac{m}{r}\left[1+\frac{2 \dot{r}}{r} \tau+\left(\frac{3 \dot{r}^{2}}{r^{2}}-\frac{\ddot{r}}{r}\right) \tau^{2}\right],
$$

To develop some easier calculations we can reject, as before, on the right hand side of expression (9) the term with $\ddot{r}$ (in fact this term is negligible and only gives terms of higher order). Hence, at this approximation, we obtain the velocity-dependent potential

$$
V \approx-\frac{m}{r}\left[1+\frac{2 \dot{r}}{r} \tau+\frac{3 \dot{r}^{2}}{r^{2}} \tau^{2}\right],
$$

In a first approximation, the delay $\tau$ must be equal to $r / c$ (the time that the field uses to goes from Mercury to the Sun at the speed of the light) according with the theories developed in [12, 13]. Introducing this expression of the delay in (10) we have:

$$
V \approx-\frac{m}{r}\left[1+\frac{2 r \dot{r}}{c r}+\frac{3 r^{2} \dot{r}^{2}}{c^{2} r^{2}}\right]
$$

On this basis, of this velocity-dependent potential function (11), the gravitational force law is given by substituting the potential function (11) into the equation:

$$
f=\frac{d}{d t}\left(\frac{\partial V}{\partial \dot{r}}\right)-\frac{\partial V}{\partial r}=-\frac{m}{r^{2}}\left(1-\frac{3 \dot{r}^{2}}{c^{2}}+\frac{6 r \ddot{r}}{c^{2}}\right) .
$$

Hence, we obtain (without fixing any parameters) the same radial force, at first orders, that gives Gerber's potential, see (2).

In fact, it is straightforward to see that, at first order, the retarded potential (7) and Gerber's potential coincide. If we develop the retarded potential (17) we have

$$
\begin{gathered}
V=-\frac{m}{r(t-\tau)} \frac{r(t)}{r(t-\tau)}=-\frac{m}{r(t)-\dot{r}(t) \tau+\ldots} \cdot \frac{r(t)}{r(t)-\dot{r}(t) \tau+\ldots} \\
=\frac{m}{r(t)\left(1-\frac{\dot{r}(t)}{r(t)} \tau+\ldots\right)} \cdot \frac{1}{1-\frac{\dot{r}(t)}{r(t)} \tau+\ldots} .
\end{gathered}
$$


Now substituting the delay $\tau=r / c$ we obtain

$$
V=-\frac{m}{r(t)\left(1-\frac{\dot{r}(t)}{c}+\ldots\right)} \cdot \frac{1}{1-\frac{\dot{r}(t)}{c}+\ldots} .
$$

Therefore, at first order, the retarded potential (7) has the form

$$
V=-\frac{m}{r(t)\left(\left(1-\frac{\dot{r}(t)}{c}\right)^{2}+\ldots\right)} .
$$

\section{Concluding Remarks}

Therefore the anomalous precession of the Mercury's perihelion is, in fact, to take into account the second order in the delay of the retarded potential (17) which is an approximation of the correct retarded potential (8). It lacks to see if the prediction for the deflection of electromagnetic waves grazing the Sun using this potential coincide with value given by General Relativity, assuming a plausible application of such potential to the propagation of electromagnetic waves. We hope to give an answer in a future work.

\section{Acknowledgements:}

The author would like to thank Prof. M. Grau from Universitat de Lleida for several useful conversations and remarks.

\section{REFERENCES}

[1] H. Behera And P.C. NAIK, A flat space-time relativistic explanation for the perihelion advance of Mercury, astro-ph/0306611, preprint, 2003.

[2] C. Chicone, What are the equations of motion of classical physics?, Can. Appl. Math. Q. 10 (2002), no. 1, 15-32.

[3] C. Chicone, S.M. Kopeikin, B. Mashhoon and D. Retzloff, Delay equations and radiation damping, Phys. Letters A 285 (2000), 17-16.

[4] C. Chicone, Inertial and slow manifolds for delay equations with small delays, J. Differential Equations 190 (2003), no. 2, 364-406.

[5] C. Chicone, Inertial flows, slow flows, and combinatorial identities for delay equations, J. Dynam. Differential Equations 16 (2004), no. 3, 805-831.

[6] A. Einstein, Erklärung der Perihelbewegung des Merkur aus der allgemeinen Relativitätstheorie (Explanation of the perihelion motion of mercury from the general theory of relativity). Königlich Preuáische Akademie der Wissenschaften, Sizungsberichte (1915), 831-839.

[7] A. Einstein, Die Grundlage der allgemeinen Relativitäetstheorie (The foundation of the general theory of relativity). Ann. Phys. 49 (1916), 769-822.

[8] F.G. Holzmuller, Z. Math. Phys. 15, 69 (1870).

[9] P. Gerber, Die räumliche und zeitliche Ausbreitung der Gravitation (Space and temporary propagation of gravitation). Z. Math. Phys. 43 (1898), 93-104.

[10] P. GERBER, Die Fortpflanzungsgeschwindigkeit der Gravitation (The propagation-velocity of gravitation). Ann. Phys. 52 (1917), 415-444. 
[11] J. Giné, On the classical descriptions of the quantum phenomena in the harmonic oscillator and in a charged particle under the coulomb force, Chaos Solitons Fractals 26 (2005), 1259-1266.

[12] J. Giné, On the origin of quantum mechanics, physics/0505181, preprint, Universitat de Lleida, 2005.

[13] J. GINÉ, On the origin of gravitational quantization: the Titius-Bode law, physics/0507072, preprint, Universitat de Lleida, 2005.

[14] P.S. De Laplace, Traite de Mécanique Céleste tome chapitre vii, §22, (1805).

[15] B. MashHoon, Gravitoelectromagnetism: A Brief Review, gr-qc/0311030, preprint, 2003.

[16] MathPages www.mathpages.com/rr/s6-02/6-02.htm

[17] MathPages www.mathpages.com/home/kmath527/kmath527.htm

[18] C. Neumann, Principles of Electrodynamics, Tübingen, 1868.

[19] S. Oppenheim Ann. Phys. 52, 415 (1917); 53, 31 \& 163 (1917); 54, 38 (1917).

[20] S. Oppenheim, Kritik des Newtoschen Gravitationsgesetzes, Encyklopädie der mathematischen Wissenschaften, vol. 6.2.2 pag. 80-158, Teuner, Leipzig.

[21] H. Poincaré, Mémoire sur les courbes définies par les équations différentielles. Journal de Mathématiques 37 (1881), 375-422; 8 (1882), 251296; Oeuvres de Henri Poincaré, vol. I, Gauthier-Villars, Paris, (1951), pp. 3-84.

[22] C.K. RAJu, The electrodymamic 2-body problem and the origin of quantum mechanics, Foundations of Physics 34 (2004), 937-962.

[23] C.K. RAJU, Time: towards a consistent theory, Kluwer academic, Dordrecht, 1994.

[24] F. Tisserand, Sur le mouvement des Planètes autour du Soleil, d'après a loi électrodynamique de Weber, Compt. Rend. Acad. Sci. (Paris) 75, 760 (1872); 110, 313 (1890).

[25] E. Whittaker, A History of the theories of ether and electriciy, vol. 2, Thomas Nelson, Edinburgh, 1953.

[26] C. M. Will, Propagation Speed of Gravity and the Relativistic Time Delay Astrophys. J. 590 (2003) 683-690.

Departament de Matemàtica, Universitat de Lleida, Av. Jaume II, 69. 25001 Lleida, SPAin

E-mail address: gine@eps.udl.es 